REVIEW

\title{
Substance misuse in the older population
}

\author{
A McGrath, P Crome, I B Crome
}

Postgrad Med J 2005;81:228-231. doi: 10.1136/pgmj.2004.023028

Substance misuse among the older population is largely overlooked and underreported. Many factors contribute to this, not least the fact that presentation may be atypical and hence easily missed by the medical practitioner. There may be many clues to its existence, provided the physician remains alert to these. Despite this it is quite comforting to know that once identified, the evidence to date suggests that older people may respond at least as well as younger people to treatment.

See end of article for authors' affiliations .....................

Correspondence to: Dr A McGrath, Springfield Unit, City General Hospital, Stoke on Trent ST4 6QG, UK: adrianmcgrath@aol.com

Submitted 14 April 2004 Accepted 28 July 2004

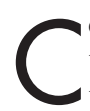
omparatively little attention has been given to the subject of substance misuse among the older population of the UK. In fact it has been referred to as the "invisible epidemic", as prevalence studies have focused mainly on younger persons. In addition, there have been variations in the terminology applied such as "older", "use", "misuse", "dependence" and relevance of these terms to an older population has been questioned. Recent surveys show that older people do indeed experience significant alcohol and drug problems, ${ }^{2}$ which may be largely under-diagnosed and under-treated. ${ }^{1}$ An atypical presentation to untrained or inexperienced practitioners may contribute to this, however, once any problem is identified the evidence suggests that there is good reason to be confident as older people will respond as well as, and perhaps even better than younger people. ${ }^{3}$

Within the older population many clinical conditions mimic substance misuse and hence without a high index of suspicion misdiagnosis is almost inevitable. In fact medical staff fail to identify two thirds of older problem drinkers, ${ }^{4}$ as both depression and dementia may be mistaken for substance misuse. Classic features of dependence, such as craving, may be absent and the older person may be in denial with not much information being offered upon direct questioning. In fact they may become very defensive and evasive. Ideally all adults over 60 should be screened for alcohol and drug misuse as part of the physical examination.

The physician should therefore be on the lookout for any of the following indications of problematic substance misuse, bearing in mind that in mild cases there may be few, if any, symptoms and signs.

Diminished psychomotor performance, amnesia and along with loss of coordination for no apparent reason may be an indicator. These factors may well contribute to falls in the older person and in such a presentation the doctor should inquire about any substances taken by the patient.
Excessive daytime drowsiness in a previously alert and active older person may well be a consequence of substance misuse and particularly if associated with persistent irritability and erratic changes in mood without obvious cause. This may be noticed and reported by close family members or friends, in fact unusual agitation and even confusion may also be apparent.

It is not unusual for a patient to present with depression, unkempt appearance, and self neglect, which may result in poor oral intake, malnutrition, and weight loss.

In addition to these, other non-specific presentations include sleep related problems such as oversleeping or insomnia, hypothermia, and possibly unexplained vomiting. In these scenarios the physician should always inquire into any prescribed drugs or non-prescribed substances taken by the person. Urinary incontinence or retention may be caused by unsuspected alcohol or substance misuse, and one should watch carefully for those at risk such as older men at times of bereavement, patients with chronic pain, and those with substance use disorders in early life or with any family history of such.

Substance misuse should be suspected from the laboratory results if liver function tests are abnormal and metabolic derangements, such as hypercholesterolaemia, hyperuricaemia, and hypoglycaemia are present. This may well be a consequence of excessive alcohol use. Additionally ethanol misuse may result in low platelets and a macrocytic anaemia, low urea and blood folate levels, with an increased $\gamma$ glutamyltransferase activity. If these abnormalities are detected then it would certainly be prudent to ask about substances taken by the patient.

Other people such as friends, employers, colleagues, relatives, or ultimately the legal system tend to identify younger substance misusers. Retirement may isolate the older person, reduce social contact, and contribute to the under-diagnosis of such patients. Physician bias can also lead to under estimating the prevalence, as the older misuser may not fit the stereotype.

A combination of willingness to consider substance misuse in the differential diagnosis, knowledge of the relevance of diagnostic classificatory systems to management, and a thorough history of alcohol, drug, prescribed medication, and over the counter medication, are more likely to lead to accurate diagnosis.

In an ideal world the best plan of action for people with complex needs related to alcohol and drug misuse is to place the patient in touch with a specialist multidisciplinary and multiagency treatment programme if appropriate. Links with 
psychiatrists and geriatricians are essential and access to the specialist unit made as efficient as possible.

Addiction services with integrated care and management are available in many NHS trusts. These new services treat drug and alcohol related problems. The principal aim being to reduce drug related harm to health and detoxification of those with substance misuse problems. They may offer diagnostic, investigative, and therapeutic screening for drug misusers.

\section{ALCOHOL}

Alcohol dependence is a disorder with bio-psycho-social influences.

The general household survey (1996) found in the UK 17\% of men and $7 \%$ of women aged 65 years or above consume more than the recommended limit and 3\%-9\% of the over $65 \mathrm{~s}$ are heavy alcohol users ( $>2$ drinks per day). Twenty per cent of the older population dependent on alcohol may misuse other drugs ${ }^{5}$ and according to the Office of National Statistics, in the 65-69 age group the prevalence of mild alcohol dependence was 17 per 1000 population, and in the $70-74$ age group it was 9 per 1000 population. ${ }^{6}$

Alcohol related mortality in Britain may be marginally higher for those born in the Caribbean than for the endogenous white population, but are substantially raised for those born in Ireland and the Indian subcontinent. ${ }^{7}$ For some years it has been recognised that up to $30 \%$ of hospitalised older patients on general medical wards may be heavy alcohol users ${ }^{5}$ and alcohol use and associated problems among nursing home residents may well exist. Up to $50 \%$ of older psychiatric in-patients have been noted to be heavy users of alcohol ${ }^{5}$ and across all ages there is a strong association between illicit drug use with alcohol dependence. ${ }^{8}$ Although mild to moderate levels of consumption may protect against dementia studies have shown alcohol use disorders closely associated with the presence of depression and are a risk factor for suicide in the elderly population. ${ }^{9}$

Early research has identified two groups of older alcohol misusers, those of early onset (survivors) and those of late onset (reactors).

Early onset alcohol misusers account for about $70 \%$ of older alcoholics, a family history of alcoholism is more prevalent in this group. They tend to have had alcohol related medical problems throughout most of their lives.

The late onset drinkers usually develop after the age of 50, often triggered by a major life stressor and higher income is a factor. The positive relation of drinking to income level has been observed in many studies of alcohol use among the elderly population ${ }^{10}$ and in addition retirement may be a risk factor for increased consumption and problems with drinking. ${ }^{11}$ This may be attributable to associated depression, lack of self esteem, or simply more time for drinking, longer opening hours, and increased availability of alcohol in supermarkets.

Because of the high prevalence of alcoholism in general hospital patients and psychiatric populations, all hospitalised patients should be asked about their pattern of alcohol use. All patients should be questioned especially in general practice and encouraged to mention their concerns.

Very brief screening methods such as CAGE and SMASTG, (see boxes $\mathrm{l}$ and 2 ) provide clinical information, but although sensitivity is variable and specifically depends on the population being screened, they can be self administered, are short, and can be incorporated into the physicians initial assessment.

Alcoholism is a significant problem in the older person and although they are unlikely to identify or report problems accurately compared with younger groups, older adults respond to treatment at least as well as younger patients in
Box 1 CAGE $^{14}-a$ useful sreening tool for detection of alcohol misuse

- C, Ever decided to cut down.

- A, Do you get annoyed when people talk to you about how much you drink.

- G, Do you have guilt feelings about your drinking.

- E, Do you ever need an eye opener to get going the next day.

terms of compliance and outcome. ${ }^{12}$ Regionally located programmes and Alcoholics Anonymous provide appropriate support.

\section{NICOTINE}

In 1995, 95000 deaths in the 65 plus age group were due to smoking in the UK. In 1996, 19\% of men aged 65-74 and 10\% of men aged $>75$ were smoking cigarettes. While in $199923 \%$ of women 65-74 and 9\% of women $>75$ smoke. ${ }^{12}$ The national statistics 2000, show that in the 65-69 age group, the number of women who had never smoked regularly was $51 \%$ compared with $30 \%$ of men.

Smoking is a major risk factor for many cardiovascular diseases, lung cancer, bladder cancer, chronic obstructive pulmonary disease, to name a few. Cessation of smoking in any age group should be encouraged. There is evidence to suggest that smokers aged 65 years or over attempting to stop smoking achieve better one year success rates than younger groups.

Randomised controlled trials show both bupropion and nicotine replacement to be effective treatments. ${ }^{13}$ Nicotine patches, lozenges, spray, and chewing gum are easily available and probably the drug of choice for older people given the side effects (for example, seizures) related to bupropion. It should be emphasised to the elderly patient by physicians that it is never too late to stop, and the range of support services offered to a determined patient as treatment of nicotine dependence is the most cost effective treatment in medicine.

Box 2 Short Michigan alcoholism screening test-geriatric version ${ }^{18}$ - a screening design specific for the older population

- Do you ever underestimate how much you drink.

- After drinking do you ever skip meals.

- Does drinking decrease shakes or tremors.

- Does alcohol make you not remember parts of the day and night.

- Do you drink to relax or calm your nerves.

- Do you drink to take your mind off problems.

- Have you ever increased your drinking after a loss in your life.

- Has a doctor or nurse said they're worried about your drinking

- Have you ever made rules to manage your drinking.

- When lonely does drinking help.

Total Smast-G score (0-10)

- >Two "yes" points to an alcohol problem 


\section{PRESCRIPTION DRUGS}

The older generation not only receive most of the prescriptions in the UK, but they are also dispensed multiple drug regimens. They take at least twice the number of medications than the young. Prescribing may be less rational, and with less adequate control and regular monitoring, particularly in institutional settings.

Because of the complexity of the medication, hoarding, and drug sharing with other people (and even with pets) it is not difficult to see how errors arise. In particular older people take many psychoactive medications, women more so, and, therefore the chance of misusing sedatives and analgesics increases significantly in the older age group.

New users of psychotropic medication are possibly likely to develop into long term users when older. The prevalence of psychotropic drug misuse is four times greater in women than men, and the risk of dependence is increased if the women happens to be widowed, less educated, of lower income, in poor health, and with reduced social support. ${ }^{14}$

Benzodiazepines increase sedation and can result in poor coordination but their role as a direct cause of falls has been controversial. A recent study showed no direct link..$^{15} 16$

The withdrawal of benzodiazepines can present with three patterns. Firstly, there may be a short level rebound state of insomnia and anxiety. Alternatively a full blown withdrawal syndrome may result with noticeable irritability, panic attacks, and palpitations for 10 to 14 days. A third pattern is that patients may experience a return of the pre-treatment anxiety. Gradually weaning patients off the culprit drugs and careful prescribing by the physician can avoid these situations. Doctors may also consider alternative medications more appropriate for the patients condition, such as good analgesic control for longstanding pain and the use of SSRIs. This may avoid the unjustified use of anxiolytics in many situations.

Pharmacists have a responsibility in stating the exact dose of medication to be taken daily by the patient when dispensing prescriptions and thus avoiding labels reading "as directed" or "as prescribed". The pharmacist is often in regular contact with such patients, is accessible, and with the extended role and involvement in clinical pharmacy this places the pharmacist in a unique position with regard to the surveillance of repeat prescriptions and supervision of over the counter sales to the public. Any potential misuse and inappropriate prescribing, particularly with regard to quantities of drugs, can be spotted. This has become much easier with the advent of computerised medication records being held at the high street chemist. The pharmacist is available for professional advice and may address any concerns patients may have.

\section{OVER THE COUNTER MEDICATION}

The problems that can arise from misuse of over the counter drugs include adverse reactions and interactions with other medicine being taken concurrently. Deregulation of many drugs from prescription only medicine to pharmacy status

Key points

- Prevalence of substance misuse in older people is underestimated.

- Physicians can help identify persons needing help, provide brief intervention at the generalist level, and make necessary referrals for specialist support.

- Available evidence shows that the older population do at least as well as younger people on treatment programmes. has meant that many drugs are now available to the public upon demand, purchased without prescription under the influence of advertising. The recommendation or giving of medicines by family or friends may exacerbate the problem. The expansion of the clinical role of the community pharmacist in the past decade should now include a comparatively thorough assessment of the patient's problems before counter prescribing of the medicine. The pharmacist in partnership with general practitioners and physicians has a responsibility in reducing misuse among the older population. The opioid based remedies have been historically prone to misuse, combinations such as paracetamol and codeine, kaolin and morphine, and codeine linctus have been available for many years. In some cases these may be taken on a protracted time scale, being purchased from different pharmacies to reduce suspicion. Registering with one particular pharmacy may help reduce this and indeed many older people like to visit the same pharmacy each time.

\section{ILLICIT DRUGS}

Opioid dependent persons do survive into old age, sometimes avoiding contact with the treatment agencies. The lifetime prevalence rates for illicit drug dependence are $17 \%$ for $18-$ $29,4 \%$ for $30-59$, and less than $1 \%$ for $>60 .{ }^{8}$ Statistics available for the older population are limited but the prevalence of drug dependence in Great Britain, within the 65-69 age group, was 4 per 1000 population for tranquillisers and in the 70-74 age group 4 per 1000 population for cannabis, and one per 1000 for tranquillisers. Life time experience of cannabis in the 65-69 age group is reported to be 7 per 1000 adult population. ${ }^{16}$

Very preliminary evidence has shown cannabis may be effective in the treatment of some long term pain. Use among the older population does occur, particularly for chronic or painful diseases such as multiple sclerosis. Pain may increase the drive to use any drug that produces euphoria and some studies have shown pain patients more likely to report severe misuse of non-prescribed opioids, sedatives, and cannabis. ${ }^{17}$

\section{CONCLUSION}

- Older people are not immune to the development of substance misuse and dependence on legal and illicit substances as well as prescribed and "over the counter medication". The nature and extent of the problem and associated psychological and physical comorbidity is greatly underestimated.

- Prescribed medicines should not be shared and relatives or friends should be aware of the risks of recommending or giving potentially addictive substances to older people.

- General and specialist physicians have a responsibility in the detection and identification of the severity of the disorder and associated complications so that appropriate generalist or specialist interventions can be efficiently accessed and implemented.

- Physicians should be aware of the local arrangements regarding referral of the older person for specialised help, and the process should be as rapid as possible.

- It should be emphasised that once recognised older addicts have a good chance of reducing or abstaining.

\footnotetext{
Authors' affiliations

A McGrath, Springfield Unit, City General Hospital, Stoke on Trent, UK

P Crome, Department of Geriatric Medicine, University of Keele, UK

I B Crome, Department of Psychiatry, University of Keele

Funding: none.
}

Conflicts of interest: none declared. 


\section{REFERENCES}

1 Widlitz M, Marin D. Substance abuse in older adults. CME 2002;57:29-34

2 Crome IB, Day E. Substance misuse and dependence, older people deserve better services. Review in Clinical Gerontology 1999:9:327-42.

3 Fitzgerald J, Mulford $\mathrm{H}$. Elderly versus younger problem drinkers treatment and recovery experiences. Br J Addict 1992;87:1281-90.

4 McInnes E, Powell J. Drug and alcohol referrals; are elderly substance abuse diagnoses and referrals being made. BMJ 1994;308:444-6.

5 Atkinson JH, Schuckit MA. Geriatric alcohol and drug misuse and abuse. Advances in Substance Abuse 1983;3:195-237.

6 Coulthard M, Farrell M, Singleton N, et al. Tobacco, alcohol and drug use and mental health. London: TSO, 2000.

7 Harrison L, Sutton M, Gardiner E. Ethnic differences in substance use and alcohol use related mortality in first generation migrants to England and Wales. Journal of Substance Use and Misuse 1997;32:849-76.

8 Hinkin C, Castellin S, Dickinson-Fuhrman E, et al. Screening for drug and alcohol abuse among older adults using modified version of CAGE. Am J Addict 2002;10:319-26.

9 Whelan G. Alcohol; a much neglected risk factor in elderly mental disorders. Current Opinions in Psychiatry 2003;16:609-14.
10 Moore A, Hays R, Greendale G, et al. Drinking habits among older persons. J Am Geriatr Soc 1999:47:412-16.

11 Ekerdt D, Delabry L, Glynn R, et al. Changes in drinking behaviour with retirement. J Stud Alcohol 1989;50:347-53.

12 Satre D, Mertens J, Arean P, et al. Contrasting outcomes of older versus middle aged and younger adult chemically dependent patients in a managed care programme. J Stud Alcohol 2003;64:520-30.

13 Hurt R, Sachs D, Glover E, et al. A comparison of sustained release bupropion and placebo for smoking cessation. N Engl J Med 1997;337:1195-202.

14 King C, Van Hasselt V, Segal D, et al. Diagnosis and assessment of substance abuse in older adults. Current strategies and issues. Addict Behav 1994; 19:41-5

15 Kelly KD, Pickett W, Yiannakoulias N, et al. Medication use and falls in community dwelling older persons. Age Ageing 2003;32:503-9.

16 Lawlor D, Patel R, Ebrahim S. Association between falls in elderly women and chronic disease and drug use. BMJ 2003;327:712-17.

17 Trafton JE, Olivia E, Horst D, et al. Humphreys K. Treatment needs associated with pain in substance use disorder patients; implications of concurrent treatment. Drug Alcohol Depend 2004;73:23-31.

18 Massachusetts Department of Public Health. Alcohol and medication issues for older adults. http://www.maclearinghouse.com/PDFs/SubstanceAbuse/ ProviderUpdate.pdf (accessed 12 Jan 2005).

\section{Clinical Evidence - Call for contributors}

Clinical Evidence is a regularly updated evidence-based journal available worldwide both as a paper version and on the internet. Clinical Evidence needs to recruit a number of new contributors. Contributors are healthcare professionals or epidemiologists with experience in evidence-based medicine and the ability to write in a concise and structured way.

Areas for which we are currently seeking authors:

- Child health: nocturnal enuresis

- Eye disorders: bacterial conjunctivitis

- Male health: prostate cancer (metastatic)

- Women's health: pre-menstrual syndrome; pyelonephritis in non-pregnant women However, we are always looking for others, so do not let this list discourage you.

Being a contributor involves:

- Selecting from a validated, screened search (performed by in-house Information Specialists) epidemiologically sound studies for inclusion.

- Documenting your decisions about which studies to include on an inclusion and exclusion form, which we keep on file.

- Writing the text to a highly structured template (about 1500-3000 words), using evidence from the final studies chosen, within 8-10 weeks of receiving the literature search.

- Working with Clinical Evidence editors to ensure that the final text meets epidemiological and style standards.

- Updating the text every six months using any new, sound evidence that becomes available. The Clinical Evidence in-house team will conduct the searches for contributors; your task is simply to filter out high quality studies and incorporate them in the existing text.

- To expand the topic to include a new question about once every 12-18 months.

If you would like to become a contributor for Clinical Evidence or require more information about what this involves please send your contact details and a copy of your CV, clearly stating the clinical area you are interested in, to Klara Brunnhuber (kbrunnhuber@ bmigroup.com).

\section{Call for peer reviewers}

Clinical Evidence also needs to recruit a number of new peer reviewers specifically with an interest in the clinical areas stated above, and also others related to general practice. Peer reviewers are healthcare professionals or epidemiologists with experience in evidence-based medicine. As a peer reviewer you would be asked for your views on the clinical relevance, validity, and accessibility of specific topics within the journal, and their usefulness to the intended audience (international generalists and healthcare professionals, possibly with limited statistical knowledge). Topics are usually 1500-3000 words in length and we would ask you to review between 2-5 topics per year. The peer review process takes place throughout the year, and our turnaround time for each review is ideally 10-14 days.

If you are interested in becoming a peer reviewer for Clinical Evidence, please complete the peer review questionnaire at www.clinicalevidence.com or contact Klara Brunnhuber (kbrunnhuber@bmjgroup.com). 